\title{
Crystalline TQPP as p-type Semiconductor: X-ray Crystallographic Investigation, OTFT Device, and Computational Analysis of Transport Properties
}

\author{
Brigitte Wex, ${ }^{\mathrm{a}}$ Ala'a O. El-Ballouli, ${ }^{\mathrm{b}}$ Antoine Vanvooren, ${ }^{\mathrm{c}}$ Ute Zschieschang, ${ }^{\mathrm{d}}$ Hagen Klauk, ${ }^{\mathrm{d}}$ \\ Jeanette A. Krause, ${ }^{\mathrm{e}}$ Jérôme Cornil, ${ }^{\mathrm{c}}$ and Bilal R. Kaafarani ${ }^{\mathrm{b}}{ }^{*}$ \\ ${ }^{a}$ Department of Natural Sciences, Lebanese American University, P.O. Box 36, Byblos, \\ Lebanon. \\ ${ }^{\mathrm{b}}$ Department of Chemistry, American University of Beirut, Beirut 1107-2020, Lebanon \\ ${ }^{c}$ Laboratory for Chemistry of Novel Materials, University of Mons, Mons, Belgium \\ ${ }^{\mathrm{d}}$ Max-Planck Institute for Solid State Research, Heisenbergstr. 1; 70569 Stuttgart, Germany \\ ${ }^{\mathrm{e}}$ Department of Chemistry, University of Cincinnati, 404 Crosley Tower, Cincinnati, OH \\ 45221, USA.
}

\footnotetext{
* To whom correspondence should be addressed. Department of Chemistry, American University of Beirut, Beirut 1107-2020, Lebanon. Phone \#: (961)3151451; Email: bilal.kaafarani@aub.edu.lb
} 


\begin{abstract}
Two

p-type semiconducting

azapyrenoacene

materials,

quinoxalino[2',3':9,10]phenanthro[4,5-abc]phenazine

(TQPP) and

$6,7,15,16-$

tetramethylquinoxalino[2',3':9,10]phenanthro[4,5-abc]phenazine $\quad\left(\mathbf{T Q P P}-\left[\mathbf{C H}_{3}\right]_{4}\right), \quad$ were characterized and were found to display high thermal stability and planar molecular geometry as revealed by single-crystal X-ray analysis. In bottom-gate p-channel organic thin-film transistors, field-effect mobilities of $2.5 \times 10^{-3} \mathrm{~cm}^{2} / \mathrm{Vs}$ and $7.5 \times 10^{-5} \mathrm{~cm}^{2} / \mathrm{Vs}$ were measured in ambient air for TQPP and TQPP- $\left[\mathrm{CH}_{3}\right]_{4}$, respectively. Computational results of reorganization energies and electronic couplings indicate larger inter and intra-columnar couplings for TQPP-[CH$]_{4}$ in comparison to TQPP and predicts the suitability of both semiconductors for hole as well as electron transporters.
\end{abstract}

\title{
Graphical Abstract
}

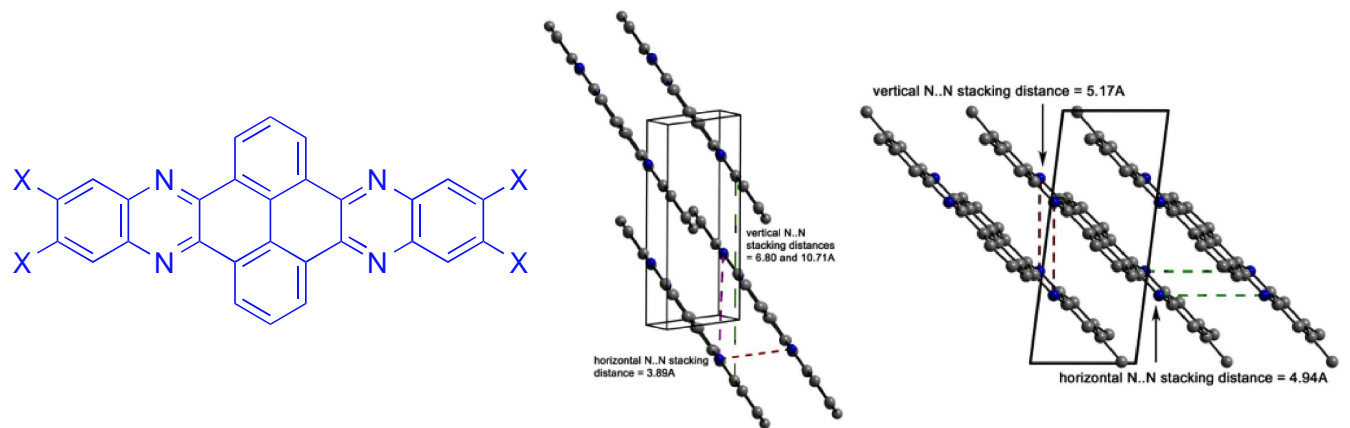

TQPP $(\mathbf{X}=\mathbf{H}): \mu=2.5 \times 10^{-3} \mathrm{~cm}^{2} / \mathrm{V} . \mathrm{s}$.

TQPP $\left(\mathbf{X}=\mathbf{C H}_{3}\right): \mu=7 \times 10^{-5} \mathrm{~cm}^{2} /$ V.s.

\section{Highlights:}

- TQPP materials with thermal stability up to $370{ }^{\circ} \mathrm{C}$ were synthesized

- TQPP and TQPP-[CH$\left.]_{3}\right]_{4}$ show interplanar stacking distances of $3.4 \AA$ using X-ray crystallography

- OTFT field-effect mobilities of $10^{-3} \mathrm{~cm}^{2} / \mathrm{V}^{-1} \mathrm{~s}^{-1}$ for TQPP and $10^{-5} \mathrm{~cm}^{2} / \mathrm{V}^{-1} \mathrm{~s}^{-1}$ TQPP$\left[\mathrm{CH}_{3}\right]_{4}$

- Modeling confirms higher electronic coupling constants for TQPP than TQPP$\left[\mathrm{CH}_{3}\right]_{4}$

Keywords: azapyrenoacene; quinoxalinophenanthrophenazine; organic thin-film transistors; field-effect mobility; electronic coupling 


\section{Introduction}

Extended, nitrogen-containing heteroacenes are derivatives of extended acene systems and have gained momentum due to their potential toward applications in liquid crystalline materials, $[1,2]$ materials with the ability to self-assemble,[3-5] as sensors as well as dyes,[69] and as n-type semiconductors.[10, 11] The latter aspect was extensively reviewed by Bunz et al.[12-14] and analyzed in a computational study by Winkler and Houk.[15] A sub-class of these materials comprise pyrene-fused pyrazaacenes, as reviewed by Mateo-Alonso, which combine the ease of synthetic versatility, using condensation between tetraketopyrene and diamine of choice, with high thermal stability and interesting materials' properties.[16, 17] Alkylthio-substituted pyrene-fused pyraazacenes TQPP-[SC$\left.{ }_{12} \mathrm{H}_{25}\right]_{4}$ have previously been studied in our group and exhibited saturation hole mobility of $\sim 10^{-3} \mathrm{~cm}^{2} \mathrm{~V}^{-1} \mathrm{~s}^{-1}$ [18] with a high tilt-angle when compared to the surface normal.[19] In this paper, we apply an efficient synthetic protocol in preparing the electron-deficient discoids, quinoxalino[2',3':9,10]phenanthro[4,5-abc]phenazine and 6,7,15,16tetramethylquinoxalino $\left[2^{\prime}, 3^{\prime}: 9,10\right]$ phenanthro[4,5-abc]phenazine, hereafter referred to as TQPP and TQPP-[CH $\left.\mathrm{CH}_{3}\right]_{4}$, respectively. Furthermore, we report a combined experimental and theoretical study of exploiting these materials as p-type semiconductors.

\section{Materials and Methods}

\subsection{Synthesis}

Tetraketopyrene (1) was prepared using a literature procedure,[20] diamines 2, e.g. 1,2phenylenediamine (2a) and 4,5-dimethylphenylenediamine (2b) were acquired from commercial sources and used as received.

\section{General procedure for the synthesis of TQPP- $[\mathrm{X}]_{4}\left(\mathrm{X}=\mathrm{H}, \mathrm{CH}_{3}\right)$}

A solution of $150 \mathrm{~mL}$ ethanol: acetic acid (1:1) was added to a mixture of tetraketopyrene $1(2.67 \mathrm{mmol})$ and diamine $2(5.34 \mathrm{mmol})$, Scheme 1 . The reaction mixture was stirred at $120{ }^{\circ} \mathrm{C}$ for $24 \mathrm{hrs}$, cooled in an ice bath and then filtered to yield a yellow solid. The solid was purified by Soxhlet extraction using toluene and was then recrystallized from 1,2dichlorobenzene to yield TQPP-[X $]_{4}\left(\mathrm{X}=\mathrm{H}, \mathrm{CH}_{3}\right)$.

Quinoxalino[2',3':9,10]phenanthro[4,5-abc]phenazine[21] (TQPP). Yellow solid. $1.00 \mathrm{~g}$ (92\%), mp>300 ${ }^{\circ} \mathrm{C}$ (Lit. $>420{ }^{\circ} \mathrm{C}$ )[21]. ${ }^{1} \mathrm{H}$ NMR (TFA-d, $\left.300 \mathrm{MHz}\right): \delta 10.077$ (d, $J=8.1 \mathrm{~Hz}$, $4 \mathrm{H}), 8.869\left(\mathrm{dd}, J_{1}=6.6 \mathrm{~Hz} ; J_{2}=3.3 \mathrm{~Hz}, 4 \mathrm{H}\right), 8.697(\mathrm{t}, J=8.1 \mathrm{~Hz}, 2 \mathrm{H}), 8.499\left(\mathrm{dd}, J_{1}=6.6\right.$ $\mathrm{Hz} ; J_{2}=3.3 \mathrm{~Hz}, 4 \mathrm{H}$ ). ${ }^{13} \mathrm{C}$ NMR (TFA-d, $75 \mathrm{MHz}$ ): $\delta$ 138.329, 137.040, 136.176, 131.335, 
130.383, 128.677, 125.198, 124.480 ppm. Anal. Calcd. for $\mathrm{C}_{28} \mathrm{H}_{14} \mathrm{~N}_{4}: \mathrm{C}, 82.74 ; \mathrm{H}, 3.47 ; \mathrm{N}$, 13.78. Found: C, 82.53; H, 3.29; N, 13.74. MALDI (m/z): [M] $]^{+}$calcd for $\mathrm{C}_{28} \mathrm{H}_{14} \mathrm{~N}_{4}, 406.12$; Found, 406.24.

6,7,15,16-Tetramethylquinoxalino $\left[2^{\prime}, 3^{\prime}: 9,10\right]$ phenanthro[4,5-abc]phenazine

(TQPP$\left.\left[\mathbf{C H}_{3}\right]_{4}\right)$. Yellow crystals. $0.75 \mathrm{~g}\left(85 \%\right.$ yield, $\mathrm{mp}>300^{\circ} \mathrm{C} .{ }^{1} \mathrm{H}(\mathrm{TFA}-\mathrm{d}, 300 \mathrm{MHz}): \delta 9.949$ (d, $J$ $=8.1 \mathrm{~Hz}, 4 \mathrm{H}), 8.619(\mathrm{t}, J=8.1 \mathrm{~Hz}, 2 \mathrm{H}), 8.542(\mathrm{~s}, 4 \mathrm{H}), 2.831(\mathrm{~s}, 12 \mathrm{H}) . \operatorname{HRMS}-\mathrm{EI}(\mathrm{m} / \mathrm{z}):[\mathrm{M}]^{+}$ calcd for $\mathrm{C}_{32} \mathrm{H}_{22} \mathrm{~N}_{4}, 462.18445$; Found, 462.18232 .

\subsection{UV-Vis Absorption}

Approx. $1 \mathrm{mg}$ of compound was weighted out and dissolved in spectrophotometry grade $o$-dichlorobenzene $(50 \mathrm{~mL})$ under heating to $70{ }^{\circ} \mathrm{C}$ and sonication. Absorbance was recorded in triplicate.

\subsection{Thermal Gravimetric Analysis (TGA)}

Thermal stability of TQPP and TQPP-[ $\left.\mathbf{C H}_{3}\right]_{\mathbf{4}}$ was probed by thermal gravimetric analysis (TGA) under nitrogen at a heating rate of $2{ }^{\circ} \mathrm{C} / \mathrm{min}$. Decomposition temperatures are given as the temperature at which $0.1 \%$ wt loss.

\subsection{Device Fabrication}

Bottom-gate, top contact OTFTs were fabricated using a heavily doped silicon wafer as the substrate and common gate electrode. The silicon surface was thermally oxidized in dry oxygen to obtain a 100-nm-thick layer of silicon dioxide. Onto the $\mathrm{SiO}_{2}$ surface, an 8-nmthick layer of aluminum oxide was deposited by atomic layer deposition at a substrate temperature of $250{ }^{\circ} \mathrm{C}$. The substrate was then immersed into a 2-propanol solution of tetradecylphosphonic acid (PCI Synthesis) for about $1 \mathrm{hr}$ to allow a 1.7-nm-thick selfassembled monolayer (SAM) of the alkylphosphonic acid to form. Thus, the gate dielectric is a combination of 100-nm-thick $\mathrm{SiO}_{2}, 8$-nm-thick $\mathrm{AlO}_{\mathrm{x}}$ and a 1.7-nm-thick SAM, having a total thickness of about $110 \mathrm{~nm}$ and a capacitance per unit area of $34 \mathrm{nF} / \mathrm{cm}^{2}$. Onto this gate dielectric, a semiconductor layer with a thickness of $30 \mathrm{~nm}$ was deposited by sublimation in vacuum. Finally, 30-nm-thick Au source and drain contacts were deposited on top of the semiconductor by thermal evaporation in vacuum through a shadow mask. All TFTs have a channel length of $100 \mu \mathrm{m}$ and a channel width of $200 \mu \mathrm{m}$. All electrical measurements were performed in ambient air at room temperature without encapsulation. The field-effect mobility was calculated in the saturation regime $\left(V_{\mathrm{DS}}=-40 \mathrm{~V}\right)$ from the slope of the forward 
sweep of the measured transfer curve $\left(\sqrt{\mathrm{I}}_{\mathrm{D}} \mathrm{vs} . \mathrm{V}_{\mathrm{GS}}\right)$, and since it varies with the gate-source voltage, it has been plotted for each data point along the transfer curve.

\subsection{Scanning Electron Microscopy}

The thin-film morphology was imaged using a Zeiss Merlin field-emission scanning electron microscope using an acceleration voltage of $5 \mathrm{kV}$, a magnification of 50,000, and an in-lens detector.

\section{Results and discussion}

\subsection{Synthesis}

Two discoids TQPP and TQPP-[CH $\left.\mathbf{C H}_{3}\right]_{4}$ were synthesized as outlined in Scheme 1. While the structure and melting point of TQPP was reported by Vollmann,[21] no further characterization data is available for this compound in the literature. Tetraketopyrene $\mathbf{1}$ was prepared using a literature procedure[20] and was condensed with the 1,2-phenylenediamine 2a or 4,5-dimethylphenylene-1,2-diamine $\mathbf{2 b}$ (both commercially available) to yield TQPP and TQPP- $\left[\mathrm{CH}_{3}\right]_{4}$, respectively. This condensation proceeded in good yields $(>80 \%)$ but gave compounds with an innate insolubility in most common organic solvents. Purification was achieved by triturating the obtained solids with boiling toluene via Soxhlet extraction for 6 hours followed by recrystallization from 1,2-dichlorobenzene. The low solubility made it difficult to collect ${ }^{13} \mathrm{C}$ NMR spectra for TQPP-[CH $\left.\mathbf{C H}_{3}\right]_{4}$ and electrochemical data on both compounds. Both, TQPP and TQPP- $\left[\mathrm{CH}_{3}\right]_{4}$ sublime so that electronic-grade purity was achieved.

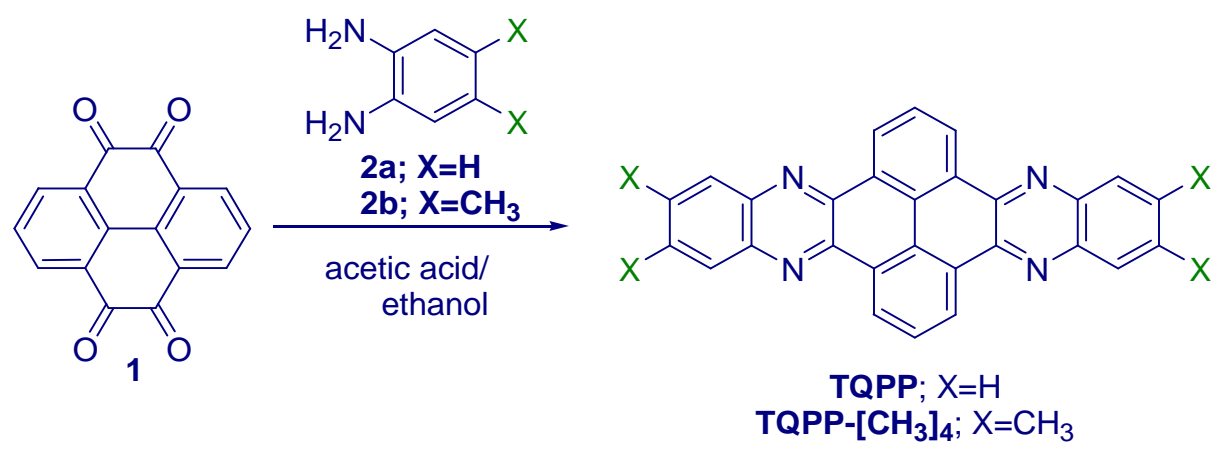

Scheme 1. Synthesis of the discoids TQPP and TQPP- $\left[\mathrm{CH}_{3}\right]_{4}$. 


\subsection{Thermal and spectroscopic properties}

Thermogravimetric analysis (TGA) showed the onset of weight loss (attributed to decomposition) of both compounds at approximately $370{ }^{\circ} \mathrm{C}$ under an air atmosphere. This observed stability is markedly higher in comparison to tetraazaoctacene, characterized by two additional fused phenyl units along the long axis of the molecule, which starts to decompose at $325{ }^{\circ} \mathrm{C}$ even under a nitrogen atmosphere.[7] The UV-vis spectra of TQPP and TQPP$\left[\mathbf{C H}_{3}\right]_{4}$ are shown in Figure 1. TQPP- $\left[\mathbf{C H}_{3}\right]_{4}$ showed characteristic absorption bands at 309, 326, 355, 376, 396, and $420 \mathrm{~nm}$. An average $3 \mathrm{meV}$ bathochromic shift is observed for the longest wavelength absorption bands. This shift is ascribed to the electron-releasing effect of the methyl groups[22] expected to lower the HOMO energy level of $\mathbf{T Q P P}-\left[\mathbf{C H}_{3}\right]_{4}$ as compared to the parent TQPP.

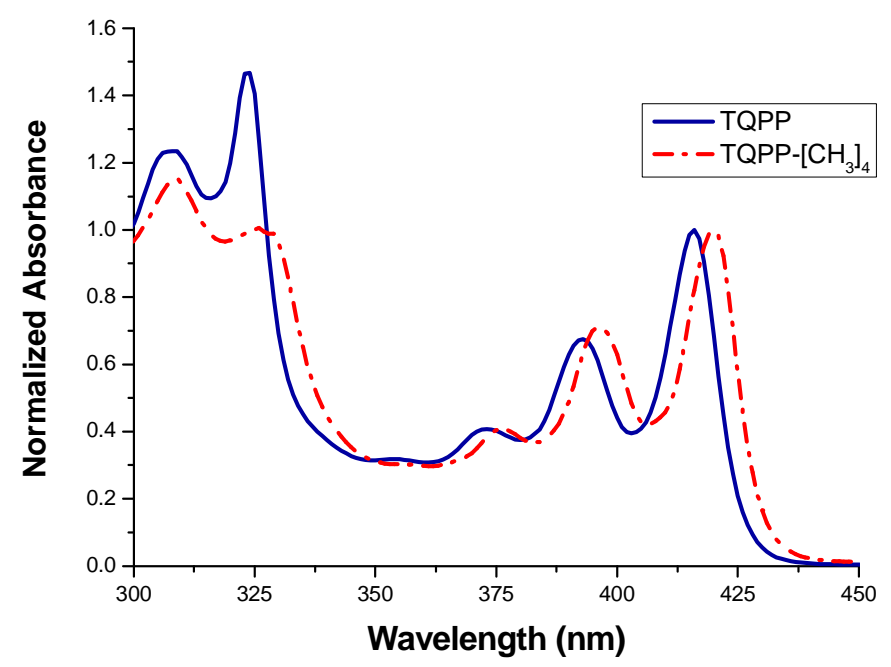

Figure 1. Absorption of TQPP and TQPP- $\left[\mathrm{CH}_{3}\right]_{4}$ recorded in 1,2-dichlorobenzene.

\subsection{Single crystal $X$-ray crystal structure analysis}

The molecular structures of TQPP and TQPP- $\left[\mathbf{C H}_{3}\right]_{4}$ were determined by single crystal X-ray crystal structure analysis. The structures are shown in the Supplementary data, Figure S1. The molecules are planar with deviations from planarity in the 0.004-0.008 $\AA$ range for the individual rings in TQPP and 0.002-0.008 $\AA$ for TQPP-[CH$]_{4}$ while the dihedral angles range from $1.11(3)-1.70(3)^{\circ}$ and $0.96(5)-1.53(5)^{\circ}$, respectively, see Supplementary data, Table S4.

Single crystal X-ray crystallography was used to study the extended structures of these materials in the solid state and to determine whether the discotic-based columns are vertical 
or inclined. Furthermore, the nature of intermolecular interactions between the discoid mesogens and the degree of aromatic overlap between the stacking molecules can be used to gain insight into their ability of transporting charge carriers along the column axis of the stacks.[23]

For TQPP and TQPP-[CH$\left.]_{3}\right]_{4}$, interplanar $\pi$-stacking distances of $3.42 \AA$ and $3.45 \AA$ were observed, respectively, which is well in agreement with the $\pi-\pi$ stacking distance of 3.3$3.6 \AA$ for TQPP-[ $\left.\mathrm{SC}_{12} \mathrm{H}_{25}\right]_{4}$.[19] Packing motives of interleaved stacked sheets for TQPP Figure 2 (left) and stacked sheets for TQPP-[C $\left.\mathbf{C H}_{3}\right]_{4}$ Figure 2 (right) were observed.
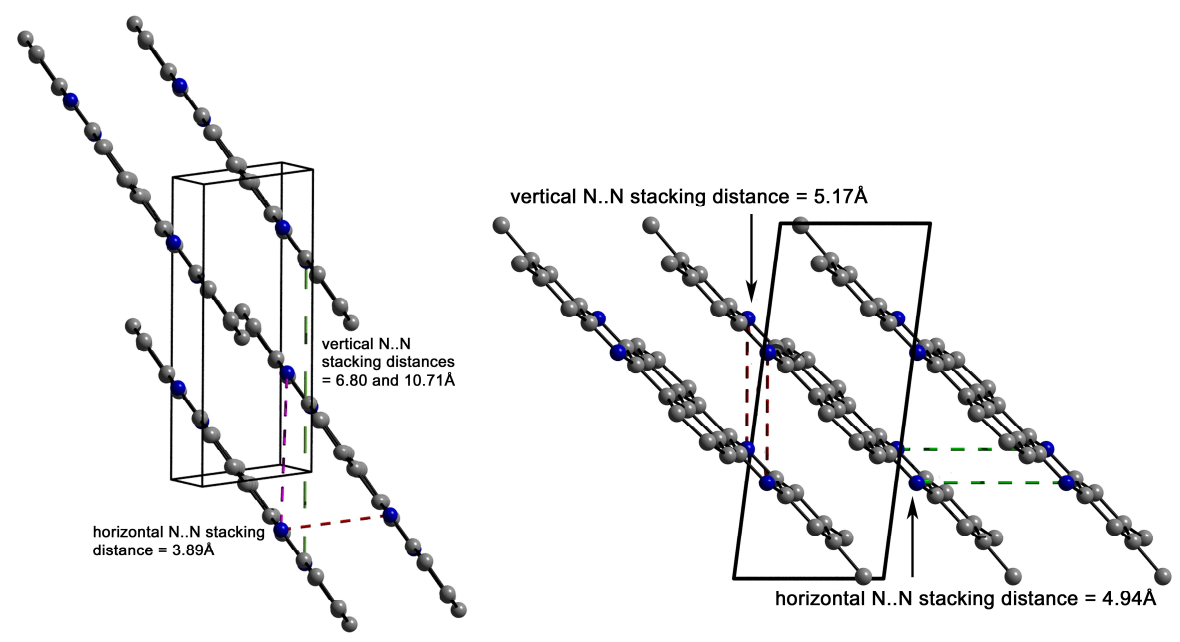

Figure 2. $\pi-$ Stacking distances for TQPP (left) and TQPP-[CH$]_{4}$ (right). The N..N interplanar distances are indicated in the figures.

\subsection{SEM of thin films}

The vacuum deposited thin-film morphology of the two semiconductors appears similar when studied by scanning electron microscopy. Only subtle differences are noted, e.g., in the grain size (i.e., the grains in the TQPP films appear to be larger than in TQPP-[C $\left.\mathbf{C H}_{3}\right]_{4}$ ), Figure 3. 

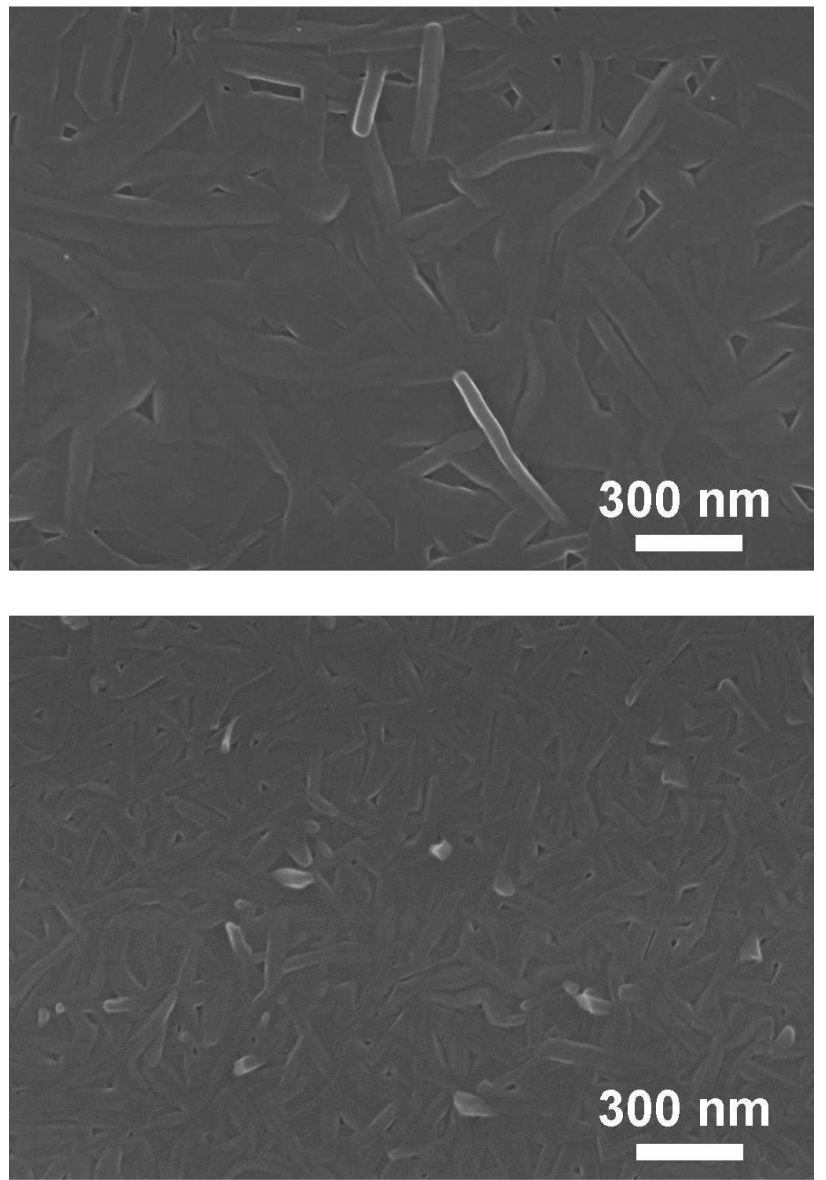

Figure 3. Scanning electron microscopy images of 30-nm-thick films of the semiconductors TQPP (top) and TQPP-[CH$]_{4}$ (bottom), vacuum-deposited onto $\mathrm{Si} / \mathrm{SiO}_{2} / \mathrm{Al}_{2} \mathrm{O}_{3} / \mathrm{SAM}$ substrates.

\subsection{Theoretical calculations}

Device performance is fundamentally influenced by the charge carrier mobility. The mobility is influenced by factors such as molecular packing, disorder, and impurities to name a few. Hence, theoretical calculations were carried out to estimate the key charge transport parameters of TQPP and TQPP-[CH $\left.\mathbf{C H}_{3}\right]_{4}$. A top view of the (columnar) structures and of the couplings between the molecules is displayed in Figures 4 and 5, respectively. 


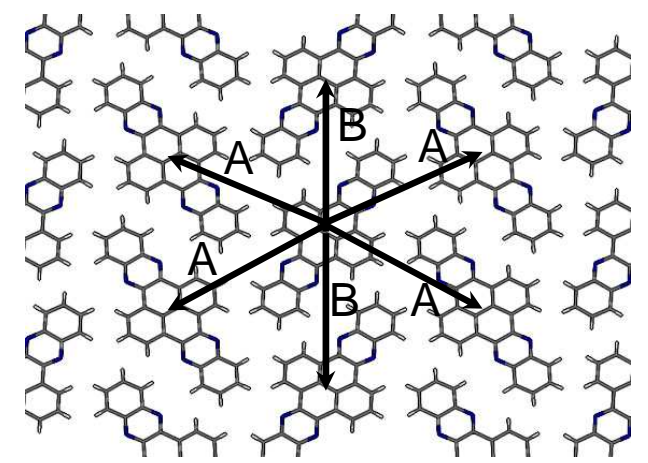

Figure 4. Inter-columnar coupling paths for TQPP.

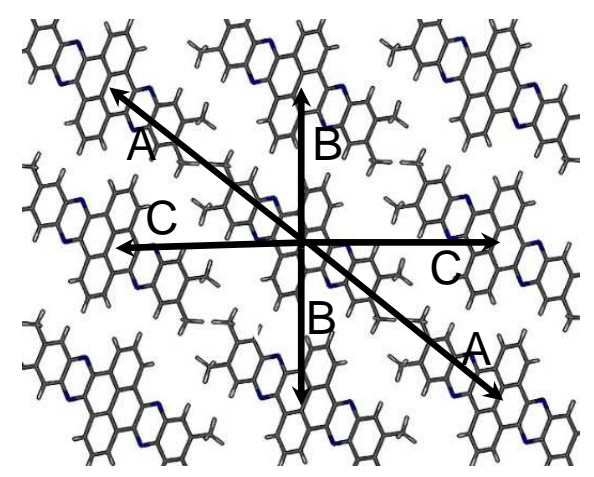

Figure 5. Inter-columnar coupling paths for TQPP- $\left[\mathrm{CH}_{3}\right]_{4}$.

The charge transfer rate between two adjacent molecules $k_{12}$ can be expressed in first approximation in the hopping regime within the semi-classical Marcus formalism as Equation (1):[24]

$$
k_{12}=\frac{2 \pi}{\hbar}\left|t_{12}\right|^{2} \frac{1}{\sqrt{4 \pi \lambda k_{B} T}} e^{-\frac{\left(\Delta G^{\circ}+\lambda\right)^{2}}{4 \lambda k_{B} T}} \text { Equation (1) }
$$

- $\quad \lambda$ is the total reorganization energy, which reflects the changes in the geometry of the molecules and the changes in the electronic and nuclear polarization of the medium between the initial and final states.

- $\Delta G^{\circ}$ is the free enthalpy of the reaction, whose value is zero for a self-exchange process, in the absence of an electric field.

- $\quad t_{12}$ is the electronic coupling between the initial and final states.

The simplest approach to calculate the transfer integral (i.e., electronic coupling) is to estimate it as half the splitting of the HOMO [LUMO] levels in a neutral dimer for hole 
[electron] transport.[25] However, for non-centrosymmetric structures, this approach is often biased by electrostatic effects, which create an initial offset of the electronic levels before they start to interact. This offset does not contribute to the transfer efficiency. In line with our recent theoretical works, we calculated the transfer integral values directly using a fragment approach at the Density Functional Theory (DFT) level using the B3LYP functional with a triple zeta polarized (TZP) basis set within the Amsterdam Density Functional (ADF) package.[26]

The estimated internal reorganization energies (at the DFT B3LYP/6-31G** level)[27] for TQPP and TQPP-[ $\left[\mathrm{CH}_{3}\right]_{4}$ are attractive compared to compounds with high charge mobilities such as oligoacenes, see Table 1.

Table 1. Calculated internal reorganization energies of discoids TQPP and TQPP- $\left[\mathrm{CH}_{3}\right]_{4}$ in comparison to representative acenes.

\begin{tabular}{|l|c|c|c|c|c|}
\hline & TQPP & $\begin{array}{c}\text { TQPP- } \\
{\left[\mathbf{C H}_{3}\right]_{4}}\end{array}$ & Tetracene[28] & Anthracene[28] & Pentacene[28] \\
\hline$\lambda_{\mathrm{i}}$ (holes), eV & 0.118 & 0.114 & 0.113 & 0.137 & 0.097 \\
\hline$\lambda_{\mathrm{i}}$ (electrons), \\
$\mathrm{eV}$
\end{tabular}

We have next calculated the electronic coupling between molecules in the same column (intra) which is expected to be the largest. This yields values of $62.4 \mathrm{meV}$ and $14.5 \mathrm{meV}$ for holes and electrons, respectively, in TQPP while values of $8.6 \mathrm{meV}$ and $18.6 \mathrm{meV}$ are obtained for TQPP- $\left[\mathrm{CH}_{3}\right]_{4}$. In contrast, the electronic coupling in tetracene, pentacene, and rubrene are on the order of 70, 85, and $83 \mathrm{meV}$, respectively.[29]

We have also estimated the electronic couplings for the major inter-columnar paths that are represented in Figures 4 and 5. Note that for each path (A, B, C), the charge can be transferred either to the molecule in the plane located above that of the initial site or to a molecule in the lower plane (i.e., to the two closest neighbors), Table 2. This leads to two possible jumps along each path (A and $A^{\prime}$ for example). The results show that the transport is mostly one-dimensional for holes in TQPP since the electronic coupling largely dominates between adjacent molecules lying in the same column. In contrast, a two-dimensional character prevails for holes in TQPP-[C $\left.\mathbf{C H}_{3}\right]_{4}$ where inter-columnar and intra-columnar hopping processes appear to be similarly efficient. 
Table 2. Electronic coupling of TQPP and TQPP- $\left[\mathrm{CH}_{3}\right]_{4}$ for the different paths.

\begin{tabular}{|c|c|c|c|}
\hline & \multicolumn{3}{|c|}{ Electronic coupling $\left(\mathrm{cm}^{-1}\right)$} \\
\hline & & HOMO & LUMO \\
\hline \multirow{5}{*}{$\stackrel{\overrightarrow{\mathrm{o}}}{\mathrm{o}}$} & Intra & 503 & 117 \\
\hline & $\mathrm{A}$ & 2 & 1 \\
\hline & $A^{\prime}$ & 9 & 10 \\
\hline & $\mathrm{B}$ & 43 & 1 \\
\hline & $\mathrm{B}^{\prime}$ & 120 & 69 \\
\hline \multirow{7}{*}{ 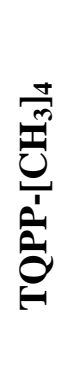 } & Intra & 69 & 150 \\
\hline & A & 29 & 29 \\
\hline & $A^{\prime}$ & 56 & 34 \\
\hline & $\mathrm{B}$ & 1 & 31 \\
\hline & $\mathrm{B}^{\prime}$ & 11 & 10 \\
\hline & $\mathrm{C}$ & 2 & 2 \\
\hline & $C^{\prime}$ & 17 & 23 \\
\hline
\end{tabular}

\subsection{Performance in OTFT device geometry}

In order to evaluate the potential of the new organic semiconductors for electronic applications experimentally, organic thin-film transistors (OTFTs) in a bottom-gate, topcontact device structure were fabricated and characterized; see Figure 6 and Table 3.

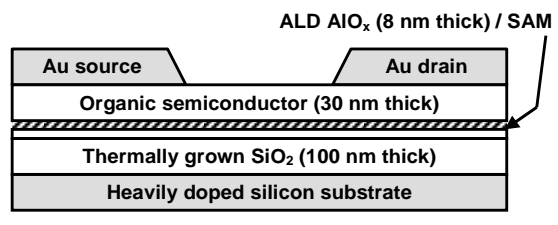

Figure 6. Schematic cross-section of the fabricated OTFTs.

Table 3. Performance of TQPP and TQPP-( $\left(\mathrm{CH}_{3}\right)_{4}$ in bottom-gate OTFTs in air.

\begin{tabular}{|c|c|c|c|}
\hline Compound & Field-effect mobility & $\begin{array}{c}\text { On/Off } \\
\text { ratio }\end{array}$ & Subthreshold swing \\
\hline TQPP & $0.0025 \mathrm{~cm}^{2} / \mathrm{Vs}$ & $10^{4}$ & $3.6 \mathrm{~V} /$ decade \\
\hline TQPP-(CH$)_{\mathbf{4}}$ & $7 \times 10^{-5} \mathrm{~cm}^{2} / \mathrm{Vs}$ & $10^{2}$ & $3.4 \mathrm{~V} /$ decade \\
\hline
\end{tabular}

The electrical measurements show that the charge-carrier mobility in thin films of TQPP is a factor of 35 larger than the charge-carrier mobility in thin films of TQPP-[ $\left[\mathbf{C H}_{3}\right]_{4}$, Figure 7. This illustrates the significant impact on the charge transport as created by the methyl 
groups at the periphery of the molecule. The semiconductor without any substitution, TQPP, gives the larger mobility $\left(0.0025 \mathrm{~cm}^{2} / \mathrm{Vs}\right)$ whilst a mobility of $7 \times 10^{-5} \mathrm{~cm}^{2} / \mathrm{Vs}$ is found in the methyl substituted semiconductor TQPP-[CH$]_{4}$.
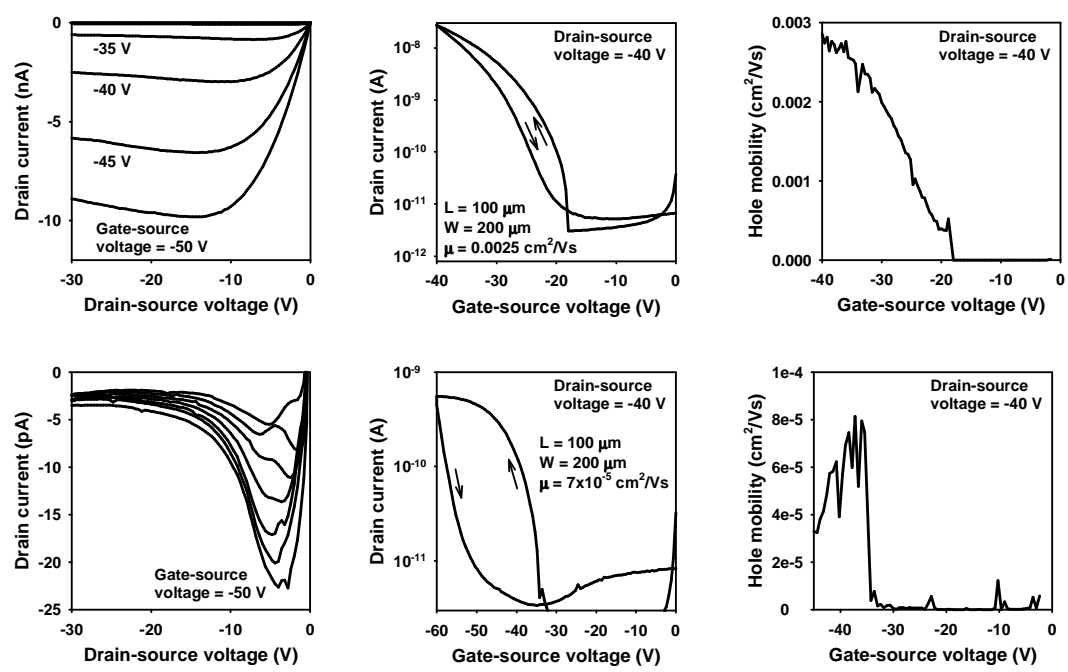

Figure 7. Measured output ( $I_{D}$ vs. $\left.V_{D S}\right)$ and transfer $\left(I_{D}\right.$ vs. $\left.V_{G S}\right)$ characteristics of the OTFTs based on TQPP (top) and TQPP-[CH$]_{4}$ (bottom) in air.

Among others, possible origins for the observed differences include different orientation of the molecules with respect to the substrate surface, grain size as well as charge transfer rates as evidenced by electronic coupling. As was observed by SEM, Figure 3, the methyl substitution has a large effect on the morphology of the semiconductor films. In particular, the size and orientation of the crystalline regions and the density of grain boundaries among others are effected.[30, 31]

In addition, the observed difference in mobilities may substantially also be rationalized by our computational simulations. According to Equation 1, the mobilities scale with the square of the electronic coupling.[32] Indeed, the highest electronic coupling for TQPP-[CH $\left.\mathbf{C H}_{3}\right]_{4}$ is 69 $\mathrm{cm}^{-1}$, a very low value compared to electronic couplings computed for TQPP $\left(503 \mathrm{~cm}^{-1}\right.$ along the stacks, and a value of $120 \mathrm{~cm}^{-1}$ for one inter-columnar coupling). Hence, the ratio between the intra-columnar couplings $(\sim 7)$ is consistent with the ratio between mobilities $(\sim 35)$.

\section{Conclusions}

In summary, both TQPP and TQPP-[ $\left.\mathrm{CH}_{3}\right]_{4}$ exhibit p-type semiconducting properties. Particularly, TQPP exhibits more favorable hole transport properties compared to TQPP$\left[\mathrm{CH}_{3}\right]_{4}$ in OFET geometry. Based on computational calculations, the electronic couplings are 
larger for TQPP than for TQPP-[CH$\left.]_{4}\right]_{4}$, especially for intra-columnar couplings. Intercolumnar couplings differ less among the two systems. TQPP shows mostly one-dimensional hole transport, while TQPP-[ $\left[\mathbf{C H}_{3}\right]_{4}$ transports holes almost equally along two directions (2D transport).

\section{Acknowledgements}

This work was supported by the Lebanese National Council for Scientific Research (CNRS), the Petroleum Research Fund (PRF) of the American Chemical Society (Grant No. 47343B10), and the Royal Society of Chemistry. The authors are grateful for this support.

Crystallographic data were collected through the SCrALS (Service Crystallography at Advanced Light Source) program at Beamline 11.3.1 at the Advanced Light Source (ALS), Lawrence Berkeley National Laboratory (supported by the U.S. Department of Energy, Office of Basic Energy Sciences, under contract No. DE-AC02-05CH11231). The work in Mons is supported by the Interuniversity Attraction Pole Programs of the Belgian Federal Science Policy Office (PAI 7/05) and by the Belgian National Fund for Scientific Research (FNRS). J.C is an FNRS Research Director. B.W. and B. R. K. thank the Arab Fund Fellowship Program for financial support.

\section{References}

[1] J. Hu, D. Zhang, S. Jin, S.Z.D. Cheng, F.W. Harris, Chem. Mater., 16 (2004) $4912-4915$. [2] S.W. Leng, L.H. Chan, J.K. Jing, J. Hu, R.M. Moustafa, R.M. Van Horn, M.J. Graham, B. Sun, M.F. Zhu, K.U. Jeong, B.R. Kaafarani, W.B. Zhang, F.W. Harris, S.Z.D. Cheng, Soft Matter, 6 (2010) 100-112.

[3] D.C. Lee, K.K. McGrath, K. Jang, Chem. Commun., (2008) 3636-3638.

[4] K.K. McGrath, K. Jang, K.A. Robins, D.C. Lee, Chem. - Eur. J., 15 (2009) 4070-4077.

[5] J. Li, P. Li, J. Wu, J. Gao, W.-W. Xiong, G. Zhang, Y. Zhao, Q. Zhang, J. Org. Chem., 79 (2014) 4438-4445.

[6] G. Li, A.P. Abiyasa, J. Gao, Y. Divayana, W. Chen, Y. Zhao, X.W. Sun, Q. Zhang, Asian J. Org. Chem., 1 (2012) 346-351. 
[7] A. Mateo-Alonso, N. Kulisic, G. Valenti, M. Marcaccio, F. Paolucci, M. Prato, Chem. Asian J., 5 (2010) 482-485.

[8] S. More, R. Bhosale, S. Choudhary, A. Mateo-Alonso, Org. Lett., 14 (2012) 4170-4173.

[9] S. More, S. Choudhary, A. Higelin, I. Krossing, M. Melle-Franco, A. Mateo-Alonso, Chem. Commun., 50 (2014) 1976-1979.

[10] B. Gao, M. Wang, Y. Cheng, L. Wang, X. Jing, F. Wang, J. Am. Chem. Soc. , 130 (2008) 8297-8306.

[11] C. Tong, W. Zhao, J. Luo, H. Mao, W. Chen, H.S.O. Chan, C. Chi, Org. Lett., 14 (2012) 494-497.

[12] U.H.F. Bunz, Chem-Eur J, 15 (2009) 6780-6789.

[13] U.H.F. Bunz, Pure Appl. Chem., 82 (2010) 953-968.

[14] U.H.F. Bunz, J.U. Engelhart, B.D. Lindner, M. Schaffroth, Angew. Chem. , 52 (2013) 3810-3821.

[15] M. Winkler, K.N. Houk, J. Am. Chem. Soc., 129 (2007) 1805-1815.

[16] A. Mateo-Alonso, Chem. Soc. Review., 43 (2014) 6311-6324.

[17] S. More, R. Bhosale, A. Mateo-Alonso, Chem-Eur J, 20 (2014) 10626-10631.

[18] B.R. Kaafarani, L.A. Lucas, B. Wex, G.E. Jabbour, Tetrahedron Lett., 48 (2007) 59955998.

[19] L.A. Lucas, D.M. DeLongchamp, L.J. Richter, R.J. Kline, D.A. Fischer, B.R. Kaafarani, G.E. Jabbour, Chem. Mater., 20 (2008) 5743-5749.

[20] J. Hu, D. Zhang, F.W. Harris, J. Org. Chem., 70 (2005) 707-708.

[21] H. Vollmann, H. Becker, M. Corell, H. Streeck, Justus Liebigs Ann. Chemie, 531 (1937) $1-159$.

[22] B.D. Koepnick, J.S. Lipscomb, D.K. Taylor, J. Phys. Chem. A, 114 (2010) 1322813233.

[23] T.L. Andresen, F.C. Krebs, N. Thorup, K. Bechgaard, Chem. Mater., 12 (2000) 24282433.

[24] R.A. Marcus, J. Chem. Phys., 43 (1965) 679-701.

[25] G. Pourtois, D. Beljonne, J. Cornil, M.A. Ratner, J.L. Brédas, J. Am. Chem. Soc., 124 (2002) 4436-4447.

[26] L. Viani, Y. Olivier, S. Athanasopoulos, D.A. da Silva, J. Hulliger, J.L. Brédas, J. Gierschner, J. Cornil, ChemPhysChem, 11 (2010) 1062-1068.

[27] A. Van Vooren, J.S. Kim, J. Cornil, ChemPhysChem, 9 (2008) 989-993.

[28] V. Coropceanu, M. Malagoli, D.A. da Silva, N.E. Gruhn, T.G. Bill, J.L. Brédas, Phys. Rev. Lett., 89 (2002).

[29] V. Coropceanu, J. Cornil, D.A. da Silva, Y. Olivier, R. Silbey, J.L. Bredas, Chem. Rev., 107 (2007) 2165-2165.

[30] H. Sirringhaus, Adv. Mater., 26 (2014) 1319-1335.

[31] C.-a. Di, Y. Liu, G. Yu, D. Zhu, Acc. Chem. Res., 42 (2009) 1573-1583.

[32] V. Lemaur, D.A. Da Silva Filho, V. Coropceanu, M. Lehmann, Y. Geerts, J. Piris, M.G. Debije, A.M. Van de Craats, K. Senthilkumar, L.D.A. Siebbeles, J.M. Warman, J.L. Brédas, J. Cornil, J. Am. Chem. Soc., 126 (2004) 3271-3279. 